\title{
Age estimations of M 31 globular clusters from their spectral energy distributions ${ }^{\star}$
}

\author{
J. Ma ${ }^{1}$, X. Zhou ${ }^{1}$, D. Burstein ${ }^{2}$, Y. Yang ${ }^{1}$, Z. Fan ${ }^{1}$, J. Chen ${ }^{1}$, Z. Jiang ${ }^{1}$, Z. Wu ${ }^{1}$, J. Wu ${ }^{1}$, and T. Zhang ${ }^{1}$ \\ 1 National Astronomical Observatories, Chinese Academy of Sciences, Beijing, 100012, PR China \\ 2 Department of Physics and Astronomy, Box 871504, Arizona State University, Tempe, AZ 85287-1504, USA \\ e-mail: majun@vega.bac.pku.edu.cn
}

Received 6 September 2005 / Accepted 15 November 2005

\begin{abstract}
This paper presents accurate spectral energy distributions (SEDs) of 15 M 31 globular clusters (GCs) confirmed by spectroscopy and/or high spatial-resolution imaging, as well as $30 \mathrm{M} 31$ globular cluster candidates detected by Mochejska et al. (1998, AcA, 48, 455). Most of these candidates have $m_{V}>18$, deeper than previous searches, and these candidates have not yet been confirmed to be globular clusters. The SEDs of these clusters and candidates are obtained as part of the BATC Multicolor Survey of the Sky, in which the spectrophotometrically-calibrated CCD images of M 31 in 13 intermediate-band filters from 4000 to $10000 \AA$ were observed. These filters are specifically designed to exclude most of the bright and variable night-sky emission lines including the $\mathrm{OH}$ forest. In comparison to the SEDs of true GCs, we find that some of the candidate objects are not GCs in M31. SED fits show that theoretical simple stellar population (SSP) models can fit the true GCs very well. We estimate the ages of these GCs by comparing with SSP models. We find that, the M 31 clusters range in age from a few ten Myr to a few Gyr old, as well as old GCs, confirming the conclusion that has been found by Barmby et al. (2000, AJ, 119, 727), Williams \& Hodge (2001, ApJ, 548, 190), Beasley et al. (2004, AJ, 128, 1623), Burstein et al. (2004, ApJ, 614, 158) and Puzia et al. (2005, A\&A, 434, 909) in their investigations of the SEDs of M 31 globular clusters.
\end{abstract}

Key words. galaxies: individual: M 31 - galaxies: star clusters - galaxies: evolution

\section{Introduction}

Galactic globular clusters are among the oldest stellar objects in the universe, provide vitally important information regarding the minimum age of the universe and the early formation history of our Galaxy. Studying the integrated properties of extragalactic globular clusters can help us understand the evolutionary history of distant galaxies (cf. Burstein et al. 2004). Globular clusters (GCs) are bright, easily identifiable stellar populations with homogeneous abundances and ages. M 31 is an ideal target for studying GCs, since it comprises the largest and nearest sample of GCs, which is more than all GCs combined in the other Local Group members (Battistini et al. 1987; Racine 1991; Harris 1991; Fusi Pecci et al. 1993).

Many GC searches in M31 have been conducted. The first catalog of M31 GC was presented by Hubble (1932), who discovered $140 \mathrm{GCs}$ with $m_{p g} \leq 18 \mathrm{mag}$. Then, a number of catalogs of GC candidates were published. Vetešnik (1962a) compiled the first major catalog, containing about $300 \mathrm{GC}$ candidates identified by the previous works (e.g., Hubble 1932; Seyfert \& Nassau 1945; Hiltner 1958;

$\star$ Table 2 is only available in electronic form at http://www. edpsciences.org
Mayall \& Eggen 1953; Kron \& Mayall 1960). Later, several other major catalogs of M31 GC candidates were compiled by Sargent et al. (1977), Crampton et al. (1985) and by the Bologna Group (Battistini et al. 1980, 1987, 1993). Although these catalogs may be fairly complete down to $V=18\left(M_{v} \sim\right.$ -6.5) (Fusi Pecci et al. 1993), recent works have searched for fainter GCs in M 31 (e.g., Mochejska et al. 1998; Barmby \& Huchra 2001).

These latter samples provide a good database for studies of M31 GCs, including their luminosity function (e.g., Aurière et al. 1992; Mochejska et al. 1998; Barmby et al. 2001), reddening and intrinsic color (e.g., Vetešnik 1962b; Bajaja \& Gergely 1977; Iye \& Richter 1985; Barmby et al. 2000; Barmby et al. 2002b), metallicities (e.g., van den Bergh 1969; Ashman \& Bird 1993; Barmby et al. 2000; Perrett et al. 2002), structure parameters (Barmby et al. 2002a) and comparisons with the Galactic GCs and M33 GCs (e.g., Hiltner 1960; Frogel et al. 1980; Reed et al. 1992; Mochejska et al. 1998). Near infrared photometry of M31 GCs has been done as well (e.g., Frogel et al. 1980; Sitko 1984; Bonoli et al. 1987, Bonoli et al. 1992; Cohen \& Matthews 1994; Barmby et al. 2000), with Galleti et al. (2004) using 2MASS data for 693 GC candidates. In a previous paper from our group, 
Jiang et al. (2003) obtained CCD multicolor photometry for 172 M 31 GCs and candidates in 13 intermediate-band filters spanning wavelengths from 4000 to $10000 \AA$.

Barmby et al. (2000), Beasley et al. (2004), Burstein et al. (2004) and Puzia et al. (2005), using SEDs, have found a set of young to intermediate-age GCs in M31, as well as the "usual" complement of GCs as old as Galactic GCs. Jiang et al. (2003) estimated ages for their M31 GCs by comparing their SEDs to those of theoretical simple stellar population (SSP) models, finding at least 8 M $31 \mathrm{GCs}$ and candidates younger than $1 \mathrm{Gyr}$. Recently, Fusi Pecci et al. (2005) found a population of 67 massive blue clusters in the disk of M31, which they interpret as globular clusters, with ages less than $\sim 2 \mathrm{Gyr}$.

M 31 was observed as part of galaxy calibration program of the Beijing-Arizona-Taiwan-Connecticut (BATC) Multicolor Sky Survey (e.g., Fan et al. 1996; Zheng et al. 1999), which has a custom-designed set of 15 intermediate-band filters to do spectrophotometry for preselected $1 \mathrm{deg}^{2}$ regions of the northern sky. In this paper, we present the SEDs for 15 M31 GCs confirmed by spectroscopy and/or high spatial-resolution imaging and $30 \mathrm{M} 31 \mathrm{GC}$ candidates detected by Mochejska et al. (1998) that lie within the BATC field of view. Details pertaining to the observations and data reduction are given in Sect. 2. Analysis of the M31 GC SEDs is reported in Sect. 3. A summary is given in Sect. 4 .

\section{Observations and data reduction}

\subsection{Sample of GCs and GC candidates}

The sample GCs and GC candidates in this paper is from Mochejska et al. (1998), who found 105 GC candidates using the data collected in the DIRECT project (Kaluzny et al. 1998; Stanek et al. 1998). DIRECT observations are done with the $1.2 \mathrm{~m}$ telescope at the F.L. Whipple Observatory. The pixel scale for the DIRECT observations is $0^{\prime} .3$. Mochejska et al. (1998) present photometry for these candidates using standard Johnson-Cousins VI filters. We were able to find 65 objects in the Mochejska et al. (1998) sample in the BATC CCD field. As 19 candidates either have bright stars nearby or have too much M 31 background, and one candidate is at the edge of the BATC CCD images in most filter bands, we do not present spectrophotometry for them (M 004, M 008, M 014, M 019, M 031, M 034, M 039, M 040, M 041, M 042, M 044, M 046, M 050, M 054, M 055, M 060, M 062, M 068, M 070 and M 072). M xxx means that this candidate is from Mochejska et al. (1998) in order to be consistent with the nomenclature of Galleti et al. (2004). Of the remaining 45 GC candidates, 15 are confirmed GCs that have also been detected by other authors (see details from Galleti et al. 2004). The SEDs of these GCs were also given by Jiang et al. (2003).

\subsection{Observations and data reduction}

The BATC survey uses a Ford Aerospace $2048 \times 2048$ CCD camera with $15 \mu \mathrm{m}$ pixel size, mounted at the focus of the 0.6/0.9m f/3 Schmidt telescope located at the Xinglong Station of the National Astronomical Observatories of China. The typical seeing of the Xinglong station is $2^{\prime \prime}$. The multicolor BATC filter system was specifically designed to avoid contamination from the brightest and most variable night sky emission lines. Our 15 intermediate-band filters cover the wavelength range from $3300 \AA$ to $1 \mu$. Spectrophotometric calibrations of these images are made using observations of four $F$ sub-dwarfs, HD 19445, HD 84937, BD $+26^{\circ} 2606$, and BD $+17^{\circ} 4708$, all taken from Oke \& Gunn (1983). Hence, our magnitudes are defined in a way similar to the spectrophotometric AB magnitude system (i.e., the Oke \& Gunn $\tilde{f}_{v}$ monochromatic system). BATC magnitudes are defined on the AB magnitude system as

$m_{\text {batc }}=-2.5 \log \tilde{F}_{v}-48.60$,

where $\tilde{F}_{v}$ is the appropriately averaged monochromatic flux in unit of erg s $\mathrm{s}^{-1} \mathrm{~cm}^{-2} \mathrm{~Hz}^{-1}$ at the effective wavelength of the specific passband. In the BATC system (Yan et al. 2000), $\tilde{F}_{v}$ is defined as

$\tilde{F}_{v}=\frac{\int \mathrm{d}(\log v) f_{v} r_{v}}{\int \mathrm{d}(\log v) r_{v}}$,

which links the magnitude to the number of photons detected by the CCD rather than to the input flux (Fukugita et al. 1996). In Eq. (2), $r_{v}$ is the system's response, $f_{v}$ is the SED of the source.

$37 \mathrm{~h}$ of images of the BATC M31 field were obtained in 13 intermediate-band filters (excluding the two bluest filters) from September 15, 1995 to December 16, 1999. Bias subtraction and flat-fielding with dome flats were done with the BATC automatic data reduction software, PIPELINE I, developed for the BATC Multicolor Sky Survey (Fan et al. 1996; Zheng et al. 1999). The dome flatfield images were taken by using a diffuser plate in front of the correcting plate of the Schmidt telescope, a flatfielding technique which has been verified with the photometry we have done on other galaxies and spectrophotometric observations (e.g., Fan et al. 1996; Zheng et al. 1999; Wu et al. 2002; Yan et al. 2000; Zhou et al. 2001). Spectrophotometric calibration of the M31 images using the Oke-Gunn standard stars is done during photometric nights (see details from Yan et al. 2000; Zhou et al. 2001).

Using the images of the standard stars observed on photometric nights, we iteratively obtain atmospheric extinction curves and variation of these extinction coefficients with time of night

(cf. Yan et al. 2000; Zhou et al. 2001):

$m_{\text {batc }}=m_{\text {inst }}+[K+\Delta K(\mathrm{UT})] X+C$,

where $X$ is the air mass and $K(\mathrm{UT})$ is the time-dependent extinction term. The instrumental magnitudes $\left(m_{\text {inst }}\right)$ of select bright, isolated and unsaturated stars on the M31 field images on photometric nights can be readily transformed to the BATC AB magnitude system ( $\left.m_{\text {batc }}\right)$. The calibrated magnitudes of these stars are then used as secondary standards to uniformly combine images from calibrated nights to those taken during non-photometric weather. Table 1 lists the parameters of the BATC filters and the statistics of observations. Column 6 of Table 1 gives the scatter, in magnitudes, for the photometric observations of the four primary standard stars in each filter. 


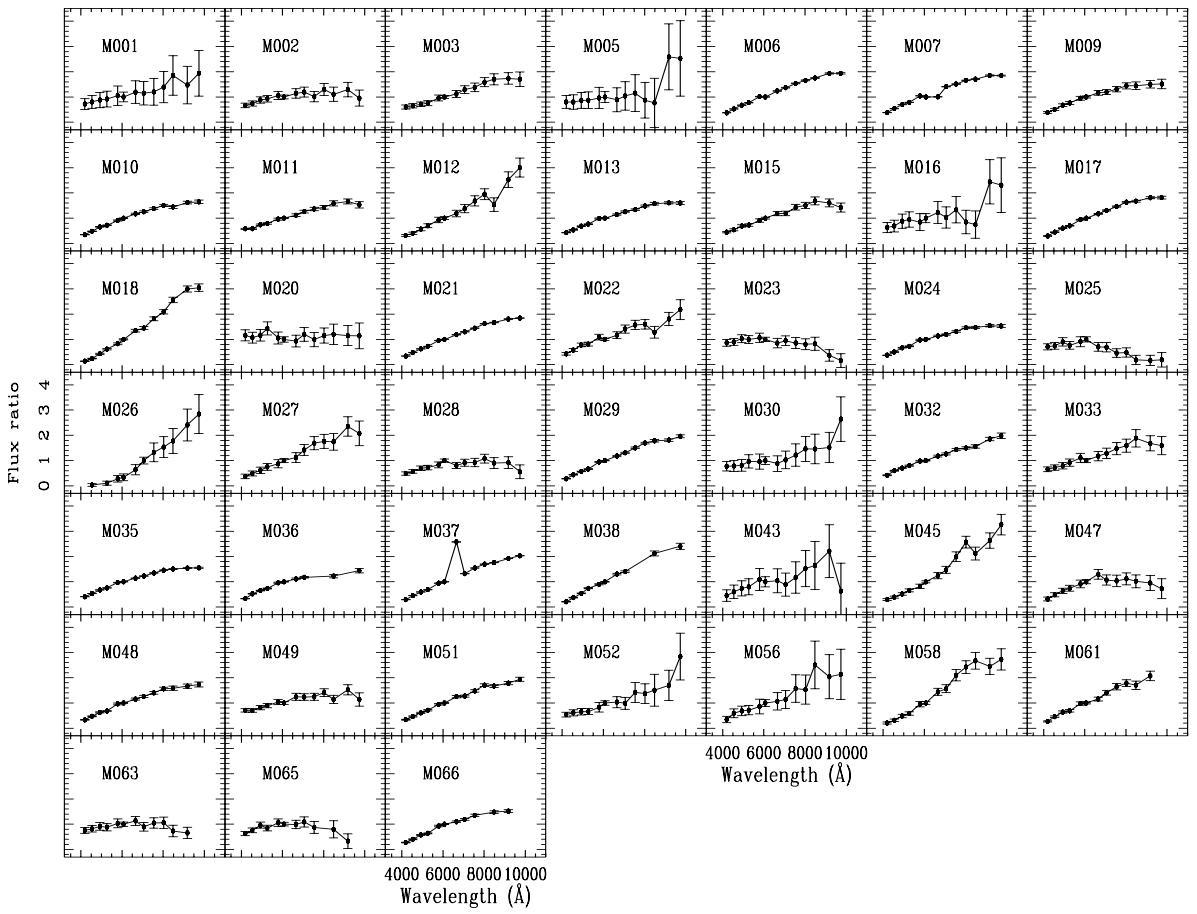

Fig. 1. Spectral energy distributions for $45 \mathrm{M} 31 \mathrm{GCs}$ and GC candidates.
Table 1. Parameters of the BATC filters and statistics of observations for M31.

\begin{tabular}{cccccc}
\hline \hline No. & Name & cw $(\AA)^{a}$ & Exp. (h) & N.img $^{b}$ & rms $^{c}$ \\
\hline 1 & BATC03 & 4210 & $01: 00$ & 03 & 0.015 \\
2 & BATC04 & 4546 & $05: 30$ & 17 & 0.009 \\
3 & BATC05 & 4872 & $03: 30$ & 11 & 0.015 \\
4 & BATC06 & 5250 & $02: 20$ & 12 & 0.006 \\
5 & BATC07 & 5785 & $02: 15$ & 07 & 0.003 \\
6 & BATC08 & 6075 & $01: 40$ & 05 & 0.003 \\
7 & BATC09 & 6710 & $00: 45$ & 03 & 0.003 \\
8 & BATC10 & 7010 & $03: 00$ & 12 & 0.008 \\
9 & BATC11 & 7530 & $02: 00$ & 06 & 0.004 \\
10 & BATC12 & 8000 & $04: 00$ & 12 & 0.003 \\
11 & BATC13 & 8510 & $01: 30$ & 05 & 0.004 \\
12 & BATC14 & 9170 & $05: 50$ & 18 & 0.003 \\
13 & BATC15 & 9720 & $04: 00$ & 12 & 0.009 \\
\hline
\end{tabular}

${ }^{a}$ Central wavelength for each BATC filter.

${ }^{b}$ Image numbers for each BATC filter.

${ }^{c}$ Calibration error, in magnitude, for each filter as obtained from the standard stars.

\subsection{Integrated photometry}

For each M 31 GC candidate, the PHOT routine in DAOPHOT (Stetson 1987) is used to obtain magnitudes. To avoid contamination from nearby objects, we adopt an aperture of $10^{\prime} .2$ corresponding to a diameter of 6 pixels on the Ford CCD. Inner and outer radii for background determination are taken at 8 to 13 pixels from the center of the GCs. Given the small aperture use for the GC observations, aperture corrections are determined as follows: we use the isolated stars to determine the magnitude difference beween photometric radii of 6 pixels and the full magnitude of these stars in each of the 13 BATC filters. The spectral energy distributions (SEDs) for $45 \mathrm{GC}$ candidates are then corrected for this difference in each filter, and these values are given in Table 2. Columns 2 to 14 give the magnitudes of the 13 BATC passbands observed. The second line for each object gives the $1-\sigma$ errors in magnitudes for the corresponding passband. The errors for each filter are given by DAOPHOT. The magnitudes of some objects in some BATC filters could not be obtained owing to low signal-to-noise ratio in these filters, or they lie at the edge of the BATC CCD images. In this table, M xxx means that this candidate is from Mochejska et al. (1998) in order to be consistent with the nomenclature of Galleti et al. (2004).

\subsection{SEDs of some objects are found not to be GCs}

Figure 1 shows the SEDs for 15 M 31 confirmed GCs and $30 \mathrm{GC}$ candidates in the 13 BATC filters. For convenience, we calculate the ratio of flux relative to the filter BATC08 $(\lambda=6075 \AA$ ) (Since M 026 has very strong emissions in red filters, we use the ratio of flux relative to the filter BATC10 $(\lambda=7010 \AA)$ in order that it can be plotted in Fig. 1.). As indicated in Section 2.1, there are 15 known GCs: M006, M007, M 010, M 011, M013, M 015, M 017, M 021, M 024, M 029, M 032, M 035, M 037, M 048, and M 051 (see details from Galleti et al. 2004). From the SEDs of these GCs, we can see that, except for M037, the SEDs of the other GCs do not vary steeply from one BATC filter band to another. In contrast, GC candidates M 005, M 016, and M 043 are likely not M31 GCs. GC candidate M 026 is very red and worthy of future study.

\section{Stellar population model and confirm GCs}

\subsection{The BCO3 models and SED fits}

Bruzual \& Charlot (2003) (hereafter BC03) provide spectral photometric properties for a wide range of stellar metallicities. 


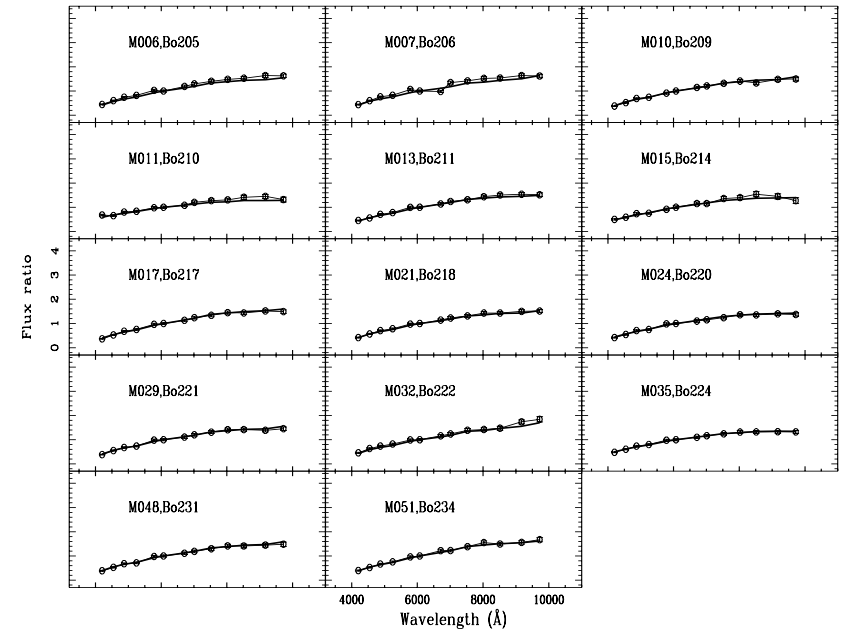

Fig. 2. Graphs of the best-fit integrated colors of SSP models plotted on top of the intrinsic integrated colors for 14 GCs. Open circles with error bars represent the intrinsic integrated color of a GC, the thick line represents the best fit of the integrated color of a SSP model. The $Y$-axis is the ratio of the flux in each filter to the flux in filter BATC08.

BC03 provide 26 SSP models (high resolution and low resolution) using the Padova 1994 evolutionary tracks. 13 of these SSP models are computed using the Chabrier (2003) IMF with lower and upper mass cutoffs $m_{L}=0.1 M_{\odot}$ and $m_{U}=100 M_{\odot}$. The other other 13 are computed using the Salpeter (1955) IMF with the same mass cutoffs. In addition, BC03 provides 26 SSP models using the Padova 2000 evolutionary tracks. However, as Bruzual \& Charlot (2003) pointed out that, the Padova 2000 models, which include more recent input physical parameters than the Padova 1994 models, tend to produce worse agreement with observed galaxy colors. These SSP models contains 221 spectra describing the spectral evolution of an SSP from 0 to $20 \mathrm{Gyr}$. The evolving spectra include the contribution of the stellar component in the range from $91 \AA$ to $160 \mu \mathrm{m}$. In this paper, we adopted the SSP models (high resolution) computing using the Padova 1994 evolutionary tracks and Salpeter (1955) IMF.

To proceed with the comparisons, we first convolve the SEDs of BC03 models with the BATC filter profiles to obtain the optical and near-infrared integrated luminosities. The integrated luminosities $L_{\lambda_{i}}(t, Z)$ of the $i$ th BATC filter can be calculated as

$L_{\lambda_{i}}(t, Z)=\frac{\int F_{\lambda}(t, Z) \varphi_{i}(\lambda) \mathrm{d} \lambda}{\int \varphi_{i}(\lambda) \mathrm{d} \lambda}$,

where $F_{\lambda}(t, Z)$ is the SEDs at age $t$ in metallicity $Z$ model, $\varphi_{i}(\lambda)$ is the response functions of the $i$ th filter of the BATC filter system $(i=3,4, \cdots, 15)$, respectively. All integrated colors of BC03 models are calculated relative to the BATC filter BATC08 $(\lambda=6075 \AA)$ :

$C_{\lambda_{i}}(t, Z)=L_{\lambda_{i}}(t, Z) / L_{6075}(t, Z)$

From this equation, we can obtain model intermediate-band colors. We use the $\chi 2$ test to examine which SED families in
Table 3. Results of SED fits between SSP models and GCs.

\begin{tabular}{cccrc}
\hline \hline Name & $\begin{array}{c}\text { Age } \\
(\mathrm{Gyr})\end{array}$ & \multicolumn{1}{c}{$\begin{array}{c}\text { Metallicity } \\
([\mathrm{Fe} / \mathrm{H}])\end{array}$} & $\begin{array}{c}\chi_{\min }^{2} \\
\text { per degree of freedom }\end{array}$ \\
\hline M006 & Bo205 & $4.73 \pm 0.65$ & $-0.81 \pm 0.02$ & 0.47 \\
M007 & Bo206 & $1.48 \pm 0.25$ & $0.00 \pm 0.07$ & 2.64 \\
M009 & Bo209 & $2.92 \pm 0.32$ & $-0.63 \pm 0.06$ & 0.64 \\
M011 & Bo210 & $1.88 \pm 0.35$ & $-1.70 \pm 0.17$ & 1.19 \\
M013 & Bo211 & $7.65 \pm 1.03$ & $-1.10 \pm 0.06$ & 0.40 \\
M015 & Bo214 & $5.78 \pm 1.12$ & $-1.35 \pm 0.26$ & 0.63 \\
M017 & Bo217 & $9.34 \pm 1.74$ & $-0.90 \pm 0.04$ & 0.75 \\
M021 & Bo218 & $2.70 \pm 0.12$ & $-0.72 \pm 0.02$ & 0.31 \\
M024 & Bo220 & $9.34 \pm 1.86$ & $-1.46 \pm 0.22$ & 0.66 \\
M029 & Bo221 & $3.04 \pm 0.32$ & $-0.72 \pm 0.02$ & 0.56 \\
M032 & Bo222 & $1.26 \pm 0.06$ & $0.00 \pm 0.06$ & 0.45 \\
M035 & Bo224 & $4.36 \pm 0.44$ & $-1.70 \pm 0.15$ & 0.37 \\
M048 & Bo231 & $2.92 \pm 0.28$ & $-0.63 \pm 0.05$ & 0.43 \\
M051 & Bo234 & $11.88 \pm 2.92$ & $-0.90 \pm 0.02$ & 0.36 \\
\hline
\end{tabular}

BC03 SSP models are most compatible with those of the observed GCs.

$\chi 2=\sum_{i=3}^{15} \frac{\left[C_{\lambda_{i}}^{\mathrm{intr}}(n)-C_{\lambda_{i}}^{\mathrm{ssp}}(t, Z)\right]^{2}}{\sigma_{i}^{2}}$

where $C_{\lambda_{i}}^{\mathrm{ssp}}(t, Z)$ represents the integrated color in the $i$ th filter of a SSP at age $t$ in a metallicity $Z$ model. $C_{\lambda_{i}}^{\text {intr }}(n)$ is the intrinsic integrated color for a GC, and

$\sigma^{2}=\sigma_{\mathrm{obs}}^{2}+\sigma_{\mathrm{mod}}^{2}$

$\sigma_{\text {obs }}^{2}$ is the observational error, and $\sigma_{\text {mod }}^{2}$ is the uncertainty from the model itself. Charlot et al. (1996) estimate the model uncertainty, $\sigma_{\text {mod }}^{2}$, by comparing the colors obtained from different stellar evolutionary tracks and spectral libraries. As Wu et al. (2005) did, we also adopted $\sigma_{\text {mod }}^{2}=0.05$ in this paper. BC03 SSP models using in this paper include six initial metallicities: $0.0001,0.0004,0.004,0.008,0.02$, and 0.05 . Spectra for other metallicities can be obtained by linear interpolation between these six base spectra.

\subsection{Reddening correction of sample GCs and candidates}

The observed colors of sample clusters or candidates are affected by two sources of reddening: the foreground extinction in the Milky Way and internal reddening in M31.

The Galactic reddening in the direction of M31 was estimated by many authors (e.g., van den Bergh 1969; McClure \& Racine 1969; Frogel et al. 1980), and similar values of the foreground color excess, $E(B-V)$, were determined, such as $E(B-V)=0.08$ given by van den Bergh (1969), 0.11 given by McClure \& Racine (1969), and 0.08 given by Frogel et al. (1980). The reddenings of 12 GCs are kindly given to us by P. Barmby. For the other objects, we accept the mean reddening, $E(B-V)=0.11$, as Fusi Pecci et al. (2005) did. The values of extinction coefficient $R_{\lambda}$ are obtained by interpolating the interstellar extinction curve of Cardelli et al. (1989). 
Table 4. Comparison of results.

\begin{tabular}{ccccc|ccc}
\hline \hline Name & $\begin{array}{c}\text { Age } \\
(\mathrm{Gyr})\end{array}$ & $\begin{array}{c}\text { Metallicity } \\
([\mathrm{Fe} / \mathrm{H}])\end{array}$ & $\begin{array}{c}\chi_{\min }^{2} \\
\text { per degree of freedom }\end{array}$ & $\begin{array}{c}\text { Age } \\
(\mathrm{Gyr})\end{array}$ & $\begin{array}{c}\text { Metallicity } \\
([\mathrm{Fe} / \mathrm{H}])\end{array}$ & $\begin{array}{c}\chi_{\text {min }}^{2} \\
\text { per degree of freedom }\end{array}$ \\
\hline M009 & Bo209 & $2.59 \pm 0.13$ & $-0.72 \pm 0.04$ & 0.74 & $2.92 \pm 0.32$ & $-0.63 \pm 0.06$ & 0.64 \\
M013 & Bo211 & $4.92 \pm 0.57$ & $-1.70 \pm 0.15$ & 0.35 & $7.65 \pm 1.03$ & $-1.10 \pm 0.06$ & 0.40 \\
M015 & Bo214 & $5.12 \pm 0.92$ & $-1.70 \pm 0.12$ & 0.60 & $5.78 \pm 1.12$ & $-1.35 \pm 0.26$ & 0.63 \\
M017 & Bo217 & $6.78 \pm 0.79$ & $-0.72 \pm 0.02$ & 0.70 & $9.34 \pm 1.74$ & $-0.90 \pm 0.04$ & 0.75 \\
M021 & Bo218 & $3.17 \pm 0.43$ & $-0.63 \pm 0.02$ & 0.30 & $2.70 \pm 0.12$ & $-0.72 \pm 0.02$ & 0.31 \\
M024 & Bo220 & $7.06 \pm 1.28$ & $-1.70 \pm 0.15$ & 0.74 & $9.34 \pm 1.86$ & $-1.46 \pm 0.22$ & 0.66 \\
\hline
\end{tabular}
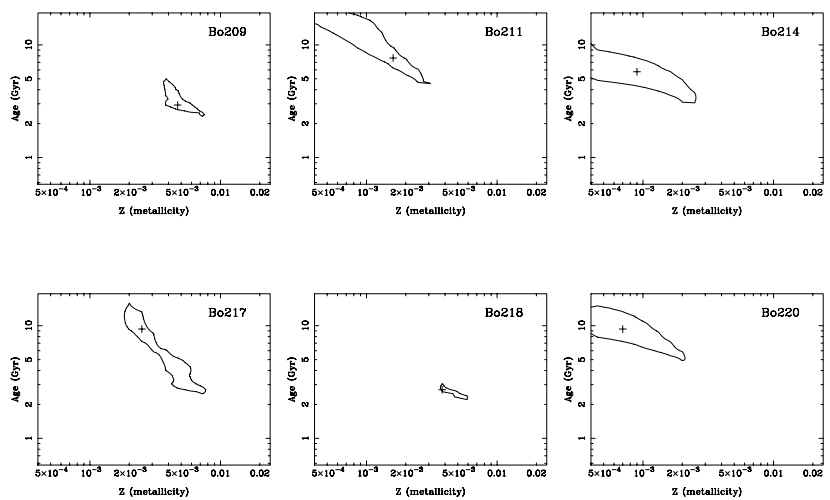

Fig. 3. Contours of $\Delta \chi 2=2.30$ for a few M 31 globular clusters.

\subsection{SED fits between SSP models and GCs}

In this paper, there are 15 known GCs confirmed by spectroscopy and/or high spatial-resolution imaging (see details from Galleti et al. 2004). We indicate that, M 037 (=Bo225 from Battistini et al. 1987), was confirmed to be a GC by spectroscopy and high spatial-resolution imaging, has an emission line in the BATC09 filter band (the central wavelength is $6710 \AA$ ) and its SEDs cannot be fit by SSP models. (It is likely that this emission line comes from the disk of M31, not from the GC itself.)

M 31 GCs generally have a metal abundance, $[\mathrm{Fe} / \mathrm{H}]$, lower than 0.3 and higher than -2.0 (Barmby et al. 2000), so, we only use models of metallicities between 0.0004 and 0.02 of BC03. Table 3 lists the results of SED fits. "Bo" means that this GC is also detected by Battistini et al. (1987). Age and metallicity mean that SEDs of GCs can best be fit by SSPs at this age in this metallicity model. Figure 2 plots the SED fits. In this figure, the open circle represents the intrinsic integrated color of the sample GCs, and the thick line represents the best fit of the integrated color of a $\mathrm{BC} 03$ model. Figure 2 shows that the SEDs of these GCs can be fit very well by BC03 models.

Extragalactic globular cluster ages are inferred from composite colors and/or spectroscopy. In the case of extragalactic GCs, young ages can be interpreted by a strong Balmer line spectrum, and/or a significant Balmer jump. The above

results can tell us that, M31 includes a population of intermediate-age GCs with ages of a few Gyr, in agreement with the conclusions of Beasley et al. (2004), Burstein et al. (2004) and Puzia et al. (2005) in their investigations of the SEDs of M 31 globular clusters.
Table 5. Results of SED fits between SSP models and GC candidates.

\begin{tabular}{lccc}
\hline \hline Name & $\begin{array}{c}\text { Age } \\
(\mathrm{Gyr})\end{array}$ & $\begin{array}{c}\text { Metallicity } \\
([\mathrm{Fe} / \mathrm{H}])\end{array}$ & $\begin{array}{c}\chi_{\min }^{2} \\
\text { per degree of freedom }\end{array}$ \\
\hline M001 & $0.52 \pm 0.04$ & $0.03 \pm 0.07$ & 0.20 \\
M002 & $0.92 \pm 0.09$ & $-1.70 \pm 0.09$ & 0.60 \\
M003 & $1.31 \pm 0.24$ & $-1.00 \pm 0.11$ & 0.91 \\
M009 & $6.78 \pm 1.29$ & $-1.70 \pm 0.10$ & 0.44 \\
M012 & $5.33 \pm 2.12$ & $0.03 \pm 0.04$ & 1.49 \\
M018 & $\ldots$ & $\ldots$ & 10.96 \\
M020 & $0.08 \pm 0.03$ & $-1.70 \pm 0.24$ & 1.06 \\
M022 & $1.48 \pm 0.16$ & $-0.44 \pm 0.22$ & 1.06 \\
M023 & $\ldots$ & $\ldots$ & 3.25 \\
M025 & $\ldots$ & $\ldots$ & 5.32 \\
M027 & $2.39 \pm 0.37$ & $0.03 \pm 0.04$ & 0.57 \\
M028 & $0.92 \pm 0.13$ & $-1.70 \pm 0.26$ & 1.66 \\
M030 & $0.02 \pm 0.00$ & $0.03 \pm 0.01$ & 0.68 \\
M033 & $1.60 \pm 0.19$ & $-1.70 \pm 0.21$ & 0.64 \\
M036 & $\ldots$ & $\ldots$ & 3.05 \\
M038 & $18.46 \pm 3.92$ & $-0.16 \pm 0.14$ & 2.35 \\
M045 & $\ldots$ & $\ldots$ & 3.19 \\
M047 & $\ldots$ & $\ldots$ & 3.24 \\
M049 & $1.08 \pm 0.07$ & $-1.70 \pm 0.13$ & 1.11 \\
M052 & $0.85 \pm 0.12$ & $0.03 \pm 0.07$ & 1.21 \\
M056 & $1.26 \pm 0.18$ & $0.03 \pm 0.23$ & 0.44 \\
M058 & $20.00 \pm 1.34$ & $0.03 \pm 0.02$ & 2.16 \\
M061 & $4.03 \pm 1.10$ & $-0.07 \pm 0.06$ & 0.74 \\
M063 & $0.01 \pm 0.00$ & $-0.44 \pm 0.12$ & 1.49 \\
M065 & $\ldots$ & $\ldots$ & 4.24 \\
M066 & $2.92 \pm 0.32$ & $-0.44 \pm 0.14$ & 1.72 \\
\hline & & &
\end{tabular}

Figure 3 shows contours of $\Delta \chi^{2}=\chi^{2}-\chi^{2}$ min in the agemetallicity plane for a few globular clusters randomly selected. It is true that the age-metallicity degeneracy exists, so it is difficult to estimate the age and metallicity simultaneously for individual clusters. However, for statistical purposes, we worked out the best fit of age and metallicity for the sample clusters from ages of 1 to $11 \mathrm{Gyr}$ and metallicities of 0.02 to $1 Z_{\odot}$.

\subsection{Comparison of results}

The observed colors of sample clusters or candidates in this paper are affected by two sources of reddening: the foreground extinction in the Milky Way and internal reddening in M31. There are few determinations of reddening for each individual cluster in M31. Barmby et al. (2000) determined reddening values for M $31 \mathrm{GCs}$ using the correlations between optical and 


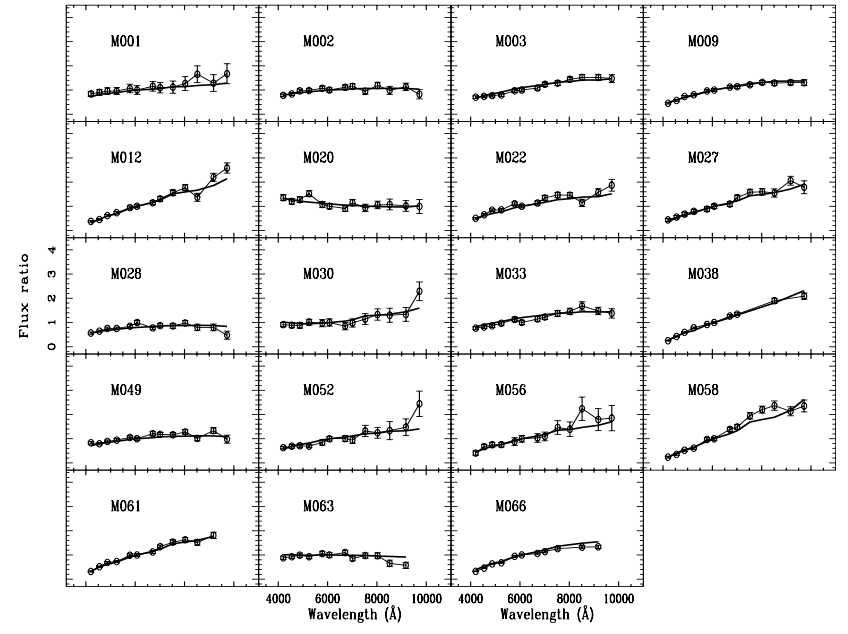

Fig. 4. Graphs of the best-fit integrated colors of SSP models plotted on top of the intrinsic integrated colors for 19 confirmed GCs. Open circles with error bars represent the intrinsic integrated color of an object, the thick line represents the best fit of the integrated color of a SSP model. The $Y$-axis is the ratio of the flux in each filter to the flux in filter BATC08.

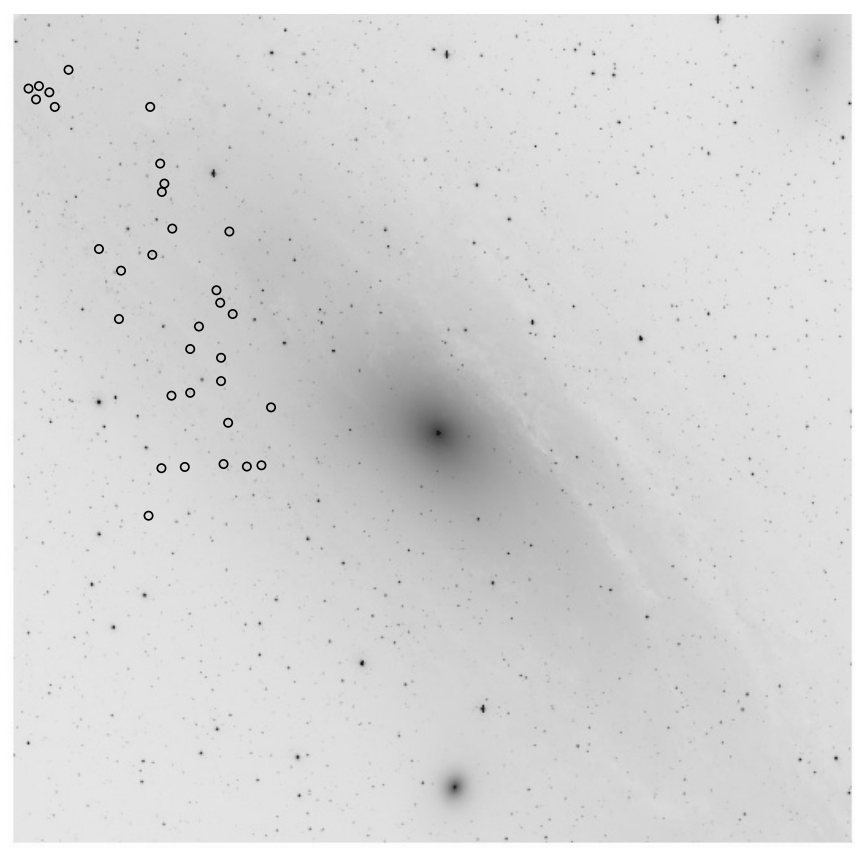

Fig. 5. The location of the clusters ontop of M31 image.

infrared colors and metallicity by defining various "reddeningfree" parameters with a large database of multicolor photometry. For showing uncertainty of the derived ages and metallicities due to the uncertain reddening, we re-estimate the ages and metallicities for the clusters in Fig. 3 as examples using the mean reddening $E(B-V)=0.11$. The results are listed in Table 4. From this table, we can see that the uncertainty of the derived ages and metallicities caused by the uncertain reddening are smaller than 3 Gyr and 0.6 dex, respectively.

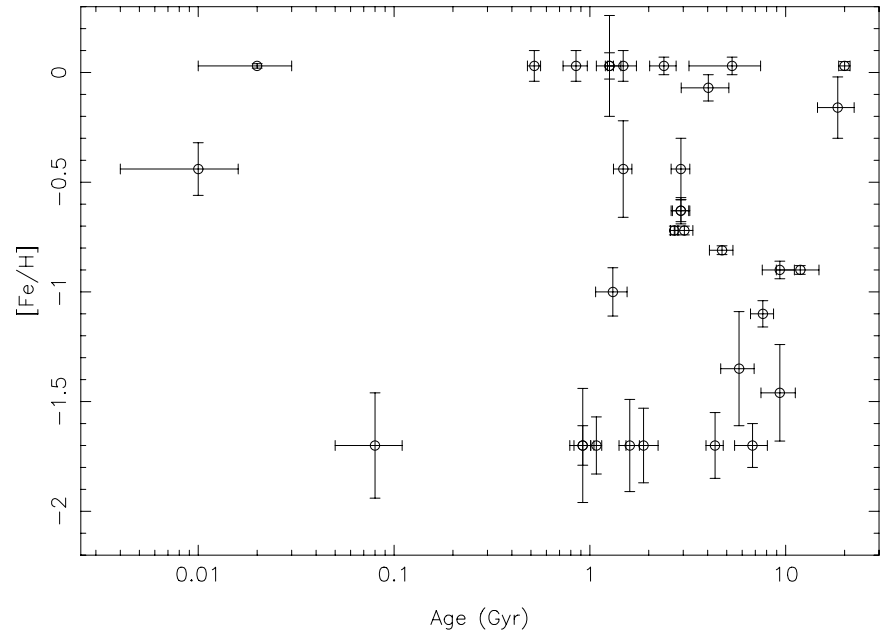

Fig. 6. Metallicity as a function of age for globular clusters of M31.

\subsection{SED fits of between SSP model and GC candidates}

We have demonstrated that the SEDs of GCs can be fit very well by SSP models. From Table 3, we find that the fittingparameter $\chi 2$ min $\leq 2.64$ for all these GCs. We take this criterion as indicating a good fit between the SSPs and the GC candidates. The results of fits between SSPs and GC candidates are listed in Table 5. If the fitting-parameter $\chi 2$ min $>2.64$, we can say that there is not an appropriate SSP to fit this candidate. From Table 5, we can see that there is 7 very young GCs, younger than 1 Gyr. Williams \& Hodge (2001) obtained deep Hubble Space Telescope (HST) photometry of individual stars and color-magnitude diagram (CMD) for four young M 31 disk clusters, subsequently identified as M 31 GCs, giving ages in the range 60-160 Myr. Beasley et al. (2004) also found an M31 GC with an age of 10-30 Myr.

Figure 4 gives the SED fits for confirmed GCs: open circles represent intrinsic integrated colors of our sample GCs, and thick lines represent the best fit of the integrated color of a SSP model. Cohen et al. (2005) present observations of 6 objects in M 31 that are alleged to be young globular clusters, and find that the four youngest of these objects are asterisms. So, we should keep in mind that the younger globular cluster candidates in this paper may be dense associations of younger stars in M31.

Combining Tables 3 and 5, we plot the location of the clusters ontop of M 31 image in Fig. 5.

\subsection{Relationship between age and metallicity}

Among the methods for age determination for GCs in the Galaxy, the most popular is the fitting of theoretical isochrones to a cluster's CMD, which requires the accurate measurement of $\Delta V$ (the difference in luminosity between the turnoff and horizontal branch), along with an estimate of $[\mathrm{Fe} / \mathrm{H}]$. Chaboyer et al. (1996) obtained ages for the 43 globular cluster using the 10 different $M_{V}(\mathrm{RR})$ relations. They plotted the age as a function of metallicity, and found clear relationship with the most metal-poor clusters being the oldest, assuming 
$M_{V}(\mathrm{RR})=0.20[\mathrm{Fe} / \mathrm{H}]+0.98$. Figure 6 plots the metallicity as a function of age for the sample globular clusters. It is clear that a relationship between age and metallicity is not found in this figure.

\section{Summary and discussion}

We have obtained SEDs of 45 faint M31 GCs and GC candidates detected by Mochejska et al. (1998) in 13 intermediate colors with the NAOC 60/90 cm Schmidt telescope. By analyzing their SEDs, 3 are likely background objects. SED fits show that theoretical simple stellar population (SSP) models can fit the true M 31 GCs very well. We confirm 20 candidates as M31 GCs, and we can estimate their ages and metallicities by comparing with SSP models. We find that these faint M 31 GCs range in age from a few ten Myr to a few Gyr old, as well as old GCs, confirming the conclusions that has been found by Barmby et al. (2000), Williams \& Hodge (2001), Beasley et al. (2004), Burstein et al. (2004) and Puzia et al. (2005).

The fact that we can find faint GCs around M 31 that have a similar wide range of ages as the brighter GCs that Beasley et al. (2004) and Burstein et al. (2004) have found is consistent with the formation scenario proposed in Burstein et al. (2004). In that scenario, these GCs come from dwarf galaxies that have been accreted by M31 in the past. Evidently, the history of accretion of its contingent of dwarf galaxies by M 31 appears to be very different from that our Galaxy has experienced.

In Table 5, there is a GC, the estimated age of which is 20 Gyr. This age only means that this GC is as old as Galactic GCs.

Acknowledgements. We would like to thank the anonymous referee for his/her insightful comments and suggestions that improved this paper very much. This work has been supported by the Chinese National Key Basic Research Science Foundation (NKBRSF TG199075402) and by the Chinese National Natural Science Foundation, Nos. 10473012 and 10573020.

\section{References}

Aurière, M., Coupinot, G., \& Hecquet, J. 1992, A\&A, 256, 95 Ashman, K. M., \& Bird, C. M. 1993, AJ, 106, 2281

Bajaja, E., \& Gergely, T. E. 1977, A\&A, 61, 229

Barmby, P., \& Huchra, J. P. 2001, AJ, 122, 2458

Barmby, P., Huchra, J., Brodie, J., et al. 2000, AJ, 119, 727

Barmby, P., Huchra, J. P., \& Brodie, J. P. 2001, AJ, 121, 1482

Barmby, P., Holland, S., \& Huchra, J. P. 2002a, AJ, 123, 1937

Barmby, P., Perrett, K. M., \& Bridges, T. J. 2002b, MNRAS, 329, 461

Battistini, P., Bònoli, F., Braccesi, A., et al. 1980, A\&AS, 42, 357

Battistini, P., Bònoli, F., Braccesi, A., et al. 1987, A\&AS, 67, 447

Battistini, P., Bònoli, F., Casavecchia, M., et al. 1993, A\&A, 272, 77

Beasley, M., et al. 2004, AJ, 128, 1623

Bonoli, F., Delpino, F., Federici, L., \& Fusi Pecci, F. 1987, A\&A, 185, 25

Bonoli, F., Delpino, F., Federici, L., Fusi Pecci, F., \& Longmore, A. J. 1992, A\&AS, 96, 163
Brown, T. M., Ferguson, H. C., Smith, E., et al. 2004, ApJ, 613, L125 Bruzual, A. G., \& Charlot, S. 2003, MNRAS, 344, 1000

Burstein, D., Li, Y., Freeman, K. C., et al. 2004, ApJ, 614, 158

Cardelli, J. A., Clayton, G. C., \& Mathis, J. S. 1989, ApJ, 345, 245

Chaboyer, B., Demarque, P., \& Sarajedini, A. A. 1996, ApJ, 459, 558 Chabrier, G. 2003, PASP, 115, 763

Charlot, S., Worthey, G., \& Bressan, A. 1996, ApJ, 457, 625

Cohen, J. G., \& Matthews, K. 1994, AJ, 108, 128

Cohen, J. G., Matthews, K., \& Cameron, P. B. 2005, ApJ, 634, L45

Crampton, D., Cowley, A. P., Schade, D., \& Chayer, P. 1985, ApJ, 288,494

Fan, X., Burstein, D., Chen, J.-S., et al. 1996, AJ, 112, 628

Frogel, J. A., Persson, S. E., \& Cohen, J. G. 1980, ApJ, 240, 785

Fukugita, M., et al. 1996, AJ, 111, 1748

Fusi Pecci, F., Cacciari, C., Federici, L., \& Pasquali, A. 1993, in The Globular Cluster-Galaxy Connection, ed. G. H. Smith, \& J. P. Brodie, 48, 410

Fusi Pecci, F., Bellazini, M., De Simone, E., \& Federici, L. 2005, AJ, 130,544

Galleti, S., Federici, L., Bellazzini, M., Fusi Pecci, F., \& Macrina, S. 2004, A\&A, 426, 917

Harris, W. E. 1991, ARA\&A, 29, 543

Hiltner, W. A. 1958, ApJ, 128, 9

Hiltner, W. A. 1960, ApJ, 131, 163

Hubble, E. P. 1932, ApJ, 76, 44

Iglesias, C. A., Rogers, F. J., \& Wilson, B. G. 1992, ApJ, 397, 717

Iye, M., \& Richter, O. G. 1985, A\&A, 144, 471

Jiang, L. H., Ma, J., Zhou, X., et al. 2003, AJ, 125, 727

Kaluzny, J., Stanek, K. Z., Krockenberger, M., et al. 1998, AJ, 115, 1016

Kron, G. E., \& Mayall, N. U. 1960, AJ, 65, 581

McClure, R. D., \& Racine, R. 1969, AJ, 74, 1000

Mayall, N. U., \& Eggen, O. J. 1953, PASP, 65, 24

Mochejska, B. J., Kaluzny, J., Krockenberger, M., Sasselov, D. D., \& Stanek, K. Z. 1998, AcA, 48, 455

Oke, J. B., \& Gunn, J. E. 1983, ApJ, 266, 713

Perrett, K. M., Bridges, T. J., Hanes, D. A., et al. 2002, AJ, 123, 2490

Puzia, T. H., Perrett, K. M., \& Bridges, T. J. 2005, A\&A, 434, 909

Racine, R. 1991, AJ, 101, 865

Reed, L. G., Harris, G. L. H., \& Harris, W. E. 1992, AJ, 103, 824

Salpeter, E. E. 1955, ApJ, 121, 161

Sargent, W. L. W., Kowal, C. T., Hartwick, F. D. A., \& van den Bergh, S. 1977, AJ, 82, 947

Seyfert, C. K., \& Nassau, J. J. 1945, ApJ, 102, 377

Sitko, M. L. 1984, ApJ, 286, 209

Stanek, K. Z., Kaluzny, J., Krockenberger, M., et al. 1998, AJ, 115, 1894

Stetson, P. B. 1987, PASP, 99, 191

van den Bergh, S. 1969, ApJS, 19, 145

Vetešnik, M. 1962a, BAC, 13, 182

Vetešnik, M. 1962b, BAC, 13, 218

Williams, B. F., \& Hodge, P. W. 2001, ApJ, 548, 190

Wu, H., Burstein, D., Deng, Z., et al. 2002, AJ, 123, 1364

Wu, H., Shao, Z. Y., Mo, H. J., Xia, X. Y., \& Deng, Z. G. 2005, ApJ, 622, 244

Yan, H. J., Burstein, D., Fan, X., et al. 2000, PASP, 112, 691

Zheng, Z. Y., Shang, Z., Su, H., et al. 1999, AJ, 117, 2757

Zhou, X., Jiang, Z. J., Xue, S. J., et al. 2001, ChJAA, 1, 372 


\section{Online Material}


J. Ma et al.: Globular clusters and candidates in M31, Online Material p 2

Table 2. SEDs of 45 GCs and GC candidates in M 31 .

\begin{tabular}{|c|c|c|c|c|c|c|c|c|c|c|c|c|c|}
\hline $\begin{array}{c}\text { Cluster } \\
\text { (1) }\end{array}$ & $\begin{array}{l}03 \\
(2)\end{array}$ & $\begin{array}{l}04 \\
\text { (3) }\end{array}$ & $\begin{array}{l}05 \\
(4)\end{array}$ & $\begin{array}{l}06 \\
(5)\end{array}$ & $\begin{array}{l}07 \\
(6)\end{array}$ & $\begin{array}{l}08 \\
(7)\end{array}$ & $\begin{array}{l}09 \\
(8)\end{array}$ & $\begin{array}{l}10 \\
(9)\end{array}$ & $\begin{array}{c}11 \\
(10)\end{array}$ & $\begin{array}{c}12 \\
(11)\end{array}$ & $\begin{array}{c}13 \\
(12)\end{array}$ & $\begin{array}{c}14 \\
(13)\end{array}$ & $\begin{array}{c}15 \\
(14)\end{array}$ \\
\hline \multirow[t]{2}{*}{ M001 } & 19.26 & 19.14 & 19.04 & 19.00 & 18.85 & 18.90 & 18.71 & 18.75 & 18.70 & 18.54 & 18.23 & 18.48 & 18.18 \\
\hline & 0.118 & 0.138 & 0.163 & 0.162 & 0.193 & 0.207 & 0.200 & 0.236 & 0.272 & 0.272 & 0.252 & 0.339 & 0.301 \\
\hline \multirow[t]{2}{*}{ M 002} & 18.53 & 18.41 & 18.22 & 18.17 & 18.03 & 18.09 & 17.94 & 17.89 & 18.07 & 17.80 & 17.98 & 17.81 & 18.14 \\
\hline & 0.067 & 0.066 & 0.068 & 0.072 & 0.082 & 0.084 & 0.094 & 0.097 & 0.139 & 0.115 & 0.191 & 0.156 & 0.280 \\
\hline \multirow[t]{2}{*}{ M 003} & 18.36 & 18.27 & 18.18 & 18.11 & 17.86 & 17.81 & 17.69 & 17.53 & 17.46 & 17.31 & 17.23 & 17.21 & 17.23 \\
\hline & 0.078 & 0.073 & 0.073 & 0.075 & 0.069 & 0.064 & 0.072 & 0.063 & 0.068 & 0.059 & 0.074 & 0.080 & 0.117 \\
\hline \multirow[t]{2}{*}{ M 005} & 19.79 & 19.79 & 19.71 & 19.70 & 19.59 & 19.55 & 19.67 & 19.50 & 19.40 & 19.70 & 19.84 & 18.52 & 18.54 \\
\hline & 0.115 & 0.153 & 0.159 & 0.179 & 0.244 & 0.231 & 0.347 & 0.357 & 0.456 & 0.646 & 1.152 & 0.317 & 0.410 \\
\hline \multirow[t]{2}{*}{ M 006} & 16.32 & 15.94 & 15.67 & 15.52 & 15.23 & 15.24 & 15.01 & 14.90 & 14.78 & 14.69 & 14.63 & 14.53 & 14.52 \\
\hline & 0.007 & 0.005 & 0.005 & 0.005 & 0.005 & 0.005 & 0.006 & 0.005 & 0.006 & 0.006 & 0.009 & 0.008 & 0.011 \\
\hline \multirow[t]{2}{*}{ M 007} & 15.92 & 15.54 & 15.25 & 15.13 & 14.82 & 14.87 & 14.86 & 14.50 & 14.42 & 14.32 & 14.29 & 14.20 & 14.20 \\
\hline & 0.005 & 0.004 & 0.004 & 0.003 & 0.004 & 0.004 & 0.005 & 0.004 & 0.005 & 0.004 & 0.007 & 0.007 & 0.008 \\
\hline \multirow[t]{2}{*}{ М 009} & 18.43 & 18.14 & 17.83 & 17.69 & 17.46 & 17.40 & 17.24 & 17.21 & 17.11 & 17.00 & 17.00 & 16.96 & 16.95 \\
\hline & 0.055 & 0.046 & 0.039 & 0.041 & 0.041 & 0.042 & 0.043 & 0.045 & 0.048 & 0.047 & 0.069 & 0.065 & 0.091 \\
\hline \multirow[t]{2}{*}{ M 010} & 17.48 & 17.11 & 16.78 & 16.68 & 16.43 & 16.32 & 16.15 & 16.08 & 15.97 & 15.88 & 15.93 & 15.80 & 15.78 \\
\hline & 0.012 & 0.009 & 0.011 & 0.010 & 0.013 & 0.011 & 0.013 & 0.014 & 0.017 & 0.017 & 0.029 & 0.023 & 0.033 \\
\hline \multirow[t]{2}{*}{ M 011} & 17.92 & 17.92 & 17.66 & 17.58 & 17.37 & 17.33 & 17.21 & 17.08 & 16.99 & 16.94 & 16.83 & 16.78 & 16.87 \\
\hline & 0.022 & 0.023 & 0.020 & 0.022 & 0.023 & 0.022 & 0.028 & 0.027 & 0.033 & 0.030 & 0.048 & 0.041 & 0.067 \\
\hline \multirow[t]{2}{*}{ M 012} & 19.38 & 19.13 & 18.76 & 18.53 & 18.22 & 18.14 & 17.96 & 17.80 & 17.58 & 17.42 & 17.67 & 17.14 & 16.95 \\
\hline & 0.071 & 0.071 & 0.061 & 0.055 & 0.064 & 0.059 & 0.060 & 0.065 & 0.071 & 0.060 & 0.131 & 0.074 & 0.077 \\
\hline \multirow[t]{2}{*}{ M 013} & 17.30 & 17.06 & 16.79 & 16.67 & 16.39 & 16.38 & 16.23 & 16.13 & 16.06 & 15.95 & 15.89 & 15.86 & 15.87 \\
\hline & 0.015 & 0.012 & 0.011 & 0.011 & 0.012 & 0.011 & 0.013 & 0.013 & 0.016 & 0.015 & 0.024 & 0.018 & 0.031 \\
\hline \multirow[t]{2}{*}{ M 015} & 18.32 & 18.11 & 17.84 & 17.79 & 17.55 & 17.44 & 17.26 & 17.25 & 17.05 & 17.00 & 16.88 & 16.93 & 17.06 \\
\hline & 0.037 & 0.037 & 0.038 & 0.038 & 0.043 & 0.038 & 0.037 & 0.043 & 0.048 & 0.047 & 0.064 & 0.067 & 0.097 \\
\hline \multirow[t]{2}{*}{ M016 } & 19.80 & 19.71 & 19.45 & 19.37 & 19.49 & 19.31 & 19.09 & 19.28 & 18.99 & 19.49 & 19.63 & 18.34 & 18.40 \\
\hline & 0.152 & 0.171 & 0.158 & 0.155 & 0.237 & 0.192 & 0.198 & 0.250 & 0.234 & 0.402 & 0.600 & 0.198 & 0.318 \\
\hline \multirow[t]{2}{*}{ M 017} & 17.52 & 17.07 & 16.74 & 16.58 & 16.26 & 16.20 & 16.02 & 15.90 & 15.78 & 15.66 & 15.64 & 15.55 & 15.55 \\
\hline & 0.014 & 0.009 & 0.007 & 0.008 & 0.010 & 0.008 & 0.009 & 0.010 & 0.011 & 0.009 & 0.015 & 0.016 & 0.025 \\
\hline \multirow[t]{2}{*}{ M 018} & 19.25 & 18.65 & 18.01 & 17.62 & 17.30 & 17.09 & 16.77 & 16.69 & 16.44 & 16.29 & 16.07 & 15.90 & 15.88 \\
\hline & 0.057 & 0.047 & 0.034 & 0.026 & 0.030 & 0.022 & 0.021 & 0.023 & 0.024 & 0.022 & 0.024 & 0.023 & 0.031 \\
\hline \multirow[t]{2}{*}{ M 020} & 18.66 & 18.75 & 18.65 & 18.42 & 18.76 & 18.82 & 18.89 & 18.62 & 18.82 & 18.66 & 18.62 & 18.66 & 18.67 \\
\hline & 0.091 & 0.099 & 0.098 & 0.080 & 0.127 & 0.116 & 0.153 & 0.131 & 0.191 & 0.166 & 0.248 & 0.259 & 0.372 \\
\hline \multirow[t]{2}{*}{ M 021} & 15.67 & 15.28 & 14.99 & 14.83 & 14.54 & 14.49 & 14.30 & 14.20 & 14.09 & 13.96 & 13.93 & 13.85 & 13.82 \\
\hline & 0.005 & 0.003 & 0.003 & 0.003 & 0.003 & 0.003 & 0.003 & 0.003 & 0.003 & 0.003 & 0.004 & 0.004 & 0.005 \\
\hline \multirow[t]{2}{*}{ M022 } & 19.52 & 19.20 & 18.88 & 18.83 & 18.51 & 18.60 & 18.43 & 18.23 & 18.11 & 18.09 & 18.33 & 17.96 & 17.76 \\
\hline & 0.066 & 0.054 & 0.044 & 0.050 & 0.057 & 0.056 & 0.067 & 0.066 & 0.071 & 0.071 & 0.142 & 0.098 & 0.142 \\
\hline \multirow[t]{2}{*}{ M 023} & 18.38 & 18.33 & 18.18 & 18.22 & 18.15 & 18.21 & 18.38 & 18.27 & 18.38 & 18.44 & 18.42 & 19.31 & 20.20 \\
\hline & 0.077 & 0.068 & 0.070 & 0.078 & 0.091 & 0.096 & 0.143 & 0.125 & 0.181 & 0.192 & 0.246 & 0.579 & 1.777 \\
\hline M 024 & 17.40 & 17.08 & 16.77 & 16.68 & 16.36 & 16.33 & 16.20 & 16.14 & 16.04 & 15.92 & 15.91 & 15.86 & 15.87 \\
\hline & 0.015 & 0.013 & 0.012 & 0.012 & 0.013 & 0.012 & 0.013 & 0.013 & 0.016 & 0.014 & 0.019 & 0.022 & 0.030 \\
\hline M 025 & 18.78 & 18.72 & 18.52 & 18.69 & 18.50 & 18.40 & 18.78 & 18.81 & 19.25 & 19.20 & 20.22 & 20.35 & 20.20 \\
\hline & 0.074 & 0.087 & 0.088 & 0.107 & 0.124 & 0.111 & 0.162 & 0.182 & 0.299 & 0.310 & 0.939 & 1.218 & 1.510 \\
\hline M 026 & $\ldots$ & 22.25 & $\ldots$ & 21.07 & 20.00 & 19.78 & 19.10 & 18.61 & 18.30 & 18.14 & 17.98 & 17.65 & 17.47 \\
\hline & $\ldots$ & 1.399 & $\ldots$ & 0.692 & 0.352 & 0.294 & 0.189 & 0.150 & 0.153 & 0.142 & 0.154 & 0.134 & 0.144 \\
\hline M 027 & 19.74 & 19.44 & 19.19 & 18.98 & 18.82 & 18.67 & 18.54 & 18.29 & 18.10 & 18.06 & 18.07 & 17.74 & 17.88 \\
\hline & 0.163 & 0.133 & 0.131 & 0.103 & 0.109 & 0.089 & 0.097 & 0.075 & 0.079 & 0.081 & 0.117 & 0.088 & 0.167 \\
\hline
\end{tabular}


Table 2. continued.

\begin{tabular}{|c|c|c|c|c|c|c|c|c|c|c|c|c|c|}
\hline $\begin{array}{c}\text { Cluster } \\
\text { (1) }\end{array}$ & $\begin{array}{l}03 \\
(2)\end{array}$ & $\begin{array}{l}04 \\
(3)\end{array}$ & $\begin{array}{l}05 \\
(4)\end{array}$ & $\begin{array}{l}06 \\
(5)\end{array}$ & $\begin{array}{l}07 \\
(6)\end{array}$ & $\begin{array}{l}08 \\
(7)\end{array}$ & $\begin{array}{l}09 \\
(8)\end{array}$ & $\begin{array}{l}10 \\
(9)\end{array}$ & $\begin{array}{c}11 \\
(10)\end{array}$ & $\begin{array}{c}12 \\
(11)\end{array}$ & $\begin{array}{c}13 \\
(12)\end{array}$ & $\begin{array}{c}14 \\
(13)\end{array}$ & $\begin{array}{c}15 \\
(14)\end{array}$ \\
\hline \multirow[t]{2}{*}{ M028 } & 19.02 & 18.84 & 18.63 & 18.59 & 18.43 & 18.23 & 18.47 & 18.33 & 18.32 & 18.15 & 18.35 & 18.33 & 18.87 \\
\hline & 0.076 & 0.073 & 0.071 & 0.071 & 0.080 & 0.067 & 0.095 & 0.098 & 0.130 & 0.107 & 0.191 & 0.204 & 0.462 \\
\hline \multirow[t]{2}{*}{ М 029} & 17.86 & 17.38 & 17.08 & 16.92 & 16.53 & 16.48 & 16.30 & 16.18 & 16.03 & 15.90 & 15.85 & 15.83 & 15.75 \\
\hline & 0.016 & 0.012 & 0.011 & 0.010 & 0.010 & 0.009 & 0.010 & 0.011 & 0.014 & 0.011 & 0.019 & 0.019 & 0.025 \\
\hline \multirow[t]{2}{*}{ M 030} & 19.19 & 19.18 & 19.15 & 18.96 & 18.97 & 18.92 & 19.06 & 18.89 & 18.70 & 18.51 & 18.51 & 18.46 & 17.86 \\
\hline & 0.099 & 0.128 & 0.147 & 0.132 & 0.173 & 0.167 & 0.209 & 0.200 & 0.228 & 0.203 & 0.269 & 0.254 & 0.196 \\
\hline \multirow[t]{2}{*}{ M 032} & 18.20 & 17.82 & 17.64 & 17.50 & 17.28 & 17.26 & 17.08 & 17.00 & 16.86 & 16.82 & 16.77 & 16.58 & 16.51 \\
\hline & 0.021 & 0.014 & 0.013 & 0.013 & 0.016 & 0.015 & 0.016 & 0.018 & 0.020 & 0.017 & 0.032 & 0.024 & 0.044 \\
\hline \multirow[t]{2}{*}{ M 033} & 18.73 & 18.62 & 18.52 & 18.36 & 18.16 & 18.27 & 18.09 & 18.00 & 17.85 & 17.76 & 17.58 & 17.70 & 17.76 \\
\hline & 0.073 & 0.074 & 0.083 & 0.077 & 0.083 & 0.089 & 0.082 & 0.081 & 0.091 & 0.090 & 0.109 & 0.105 & 0.156 \\
\hline \multirow[t]{2}{*}{ M 035} & 16.22 & 15.92 & 15.67 & 15.54 & 15.28 & 15.24 & 15.10 & 15.02 & 14.92 & 14.83 & 14.80 & 14.77 & 14.76 \\
\hline & 0.005 & 0.004 & 0.003 & 0.003 & 0.004 & 0.003 & 0.004 & 0.004 & 0.005 & 0.004 & 0.007 & 0.007 & 0.010 \\
\hline \multirow[t]{2}{*}{ M 036} & 18.40 & 17.92 & 17.68 & 17.55 & 17.26 & 17.21 & 17.09 & 17.04 & $\ldots$ & $\ldots$ & 16.99 & $\ldots$ & 16.82 \\
\hline & 0.023 & 0.017 & 0.010 & 0.012 & 0.014 & 0.012 & 0.015 & 0.015 & $\ldots$ & $\ldots$ & 0.047 & $\ldots$ & 0.054 \\
\hline \multirow[t]{2}{*}{ M 037} & 15.17 & 14.69 & 14.40 & 14.24 & 13.89 & 13.83 & 12.80 & 13.53 & 13.36 & 13.26 & 13.22 & 13.12 & 13.06 \\
\hline & 0.004 & 0.003 & 0.003 & 0.002 & 0.002 & 0.002 & 0.001 & 0.002 & 0.002 & 0.002 & 0.003 & 0.002 & 0.003 \\
\hline \multirow[t]{2}{*}{ М 038} & 19.13 & 18.51 & 18.10 & 17.76 & 17.55 & 17.44 & 17.15 & 17.07 & $\ldots$ & $\ldots$ & 16.62 & $\ldots$ & 16.48 \\
\hline & 0.038 & 0.028 & 0.013 & 0.013 & 0.017 & 0.012 & 0.015 & 0.013 & $\ldots$ & $\ldots$ & 0.038 & $\ldots$ & 0.044 \\
\hline \multirow[t]{2}{*}{ M 043} & 20.57 & 20.25 & 20.03 & 19.94 & 19.61 & 19.70 & 19.66 & 19.83 & 19.53 & 19.24 & 19.16 & 18.84 & 20.21 \\
\hline & 0.329 & 0.252 & 0.225 & 0.212 & 0.213 & 0.218 & 0.277 & 0.344 & 0.359 & 0.291 & 0.411 & 0.298 & 1.696 \\
\hline \multirow[t]{2}{*}{ M 045} & 19.82 & 19.51 & 19.21 & 18.95 & 18.71 & 18.50 & 18.26 & 18.08 & 17.75 & 17.47 & 17.68 & 17.44 & 17.21 \\
\hline & 0.087 & 0.067 & 0.060 & 0.049 & 0.067 & 0.048 & 0.058 & 0.056 & 0.058 & 0.049 & 0.083 & 0.078 & 0.088 \\
\hline \multirow[t]{2}{*}{ M 047} & 19.81 & 19.35 & 19.04 & 18.89 & 18.67 & 18.57 & 18.29 & 18.50 & 18.53 & 18.45 & 18.55 & 18.62 & 18.92 \\
\hline & 0.172 & 0.090 & 0.089 & 0.090 & 0.096 & 0.084 & 0.076 & 0.117 & 0.153 & 0.146 & 0.186 & 0.253 & 0.506 \\
\hline \multirow[t]{2}{*}{ М 048} & 18.14 & 17.76 & 17.45 & 17.36 & 16.98 & 16.94 & 16.79 & 16.70 & 16.58 & 16.46 & 16.44 & 16.39 & 16.35 \\
\hline & 0.033 & 0.023 & 0.019 & 0.020 & 0.021 & 0.018 & 0.020 & 0.020 & 0.025 & 0.025 & 0.041 & 0.037 & 0.046 \\
\hline \multirow[t]{2}{*}{ М 049} & 18.40 & 18.41 & 18.25 & 18.15 & 17.99 & 18.03 & 17.79 & 17.80 & 17.79 & 17.66 & 17.89 & 17.57 & 17.89 \\
\hline & 0.049 & 0.055 & 0.056 & 0.050 & 0.054 & 0.054 & 0.066 & 0.062 & 0.070 & 0.066 & 0.102 & 0.090 & 0.198 \\
\hline \multirow[t]{2}{*}{ M 051} & 17.67 & 17.33 & 17.03 & 16.86 & 16.56 & 16.50 & 16.25 & 16.23 & 16.07 & 15.92 & 15.94 & 15.87 & 15.78 \\
\hline & 0.046 & 0.030 & 0.027 & 0.020 & 0.019 & 0.018 & 0.020 & 0.015 & 0.015 & 0.015 & 0.017 & 0.019 & 0.029 \\
\hline \multirow[t]{2}{*}{ M 052} & 20.16 & 20.02 & 19.95 & 19.94 & 19.69 & 19.49 & 19.45 & 19.50 & 19.11 & 19.15 & 19.05 & 18.92 & 18.36 \\
\hline & 0.086 & 0.122 & 0.120 & 0.101 & 0.144 & 0.098 & 0.133 & 0.167 & 0.206 & 0.210 & 0.356 & 0.287 & 0.256 \\
\hline \multirow[t]{2}{*}{ M 056} & 20.50 & 19.91 & 19.75 & 19.71 & 19.51 & 19.35 & 19.28 & 19.20 & 18.86 & 18.89 & 18.35 & 18.57 & 18.52 \\
\hline & 0.236 & 0.167 & 0.146 & 0.131 & 0.216 & 0.149 & 0.198 & 0.189 & 0.228 & 0.261 & 0.258 & 0.316 & 0.357 \\
\hline \multirow[t]{2}{*}{ M 058} & 20.10 & 19.64 & 19.17 & 18.97 & 18.43 & 18.38 & 17.99 & 17.90 & 17.58 & 17.42 & 17.32 & 17.41 & 17.29 \\
\hline & 0.160 & 0.118 & 0.087 & 0.071 & 0.064 & 0.057 & 0.049 & 0.053 & 0.059 & 0.055 & 0.078 & 0.086 & 0.109 \\
\hline \multirow[t]{2}{*}{ M 061} & 19.54 & 18.97 & 18.61 & 18.52 & 18.15 & 18.12 & 17.96 & 17.75 & 17.58 & 17.49 & 17.54 & 17.33 & $\ldots$ \\
\hline & 0.068 & 0.042 & 0.039 & 0.037 & 0.039 & 0.038 & 0.035 & 0.035 & 0.045 & 0.043 & 0.069 & 0.062 & $\ldots$ \\
\hline \multirow[t]{2}{*}{ M 063} & 18.44 & 18.34 & 18.23 & 18.27 & 18.09 & 18.13 & 17.99 & 18.25 & 18.08 & 18.06 & 18.47 & 18.59 & $\ldots$ \\
\hline & 0.078 & 0.077 & 0.086 & 0.083 & 0.091 & 0.092 & 0.080 & 0.115 & 0.119 & 0.137 & 0.248 & 0.276 & $\cdots$ \\
\hline M 065 & 19.41 & 19.21 & 18.96 & 19.09 & 18.84 & 18.91 & 18.92 & 18.82 & 19.06 & $\ldots$ & 19.16 & 20.13 & $\cdots$ \\
\hline & 0.065 & 0.055 & 0.058 & 0.060 & 0.075 & 0.078 & 0.104 & 0.122 & 0.220 & $\ldots$ & 0.389 & 0.884 & $\ldots$ \\
\hline M 066 & 18.14 & 17.72 & 17.33 & 17.22 & 16.82 & 16.74 & 16.64 & 16.55 & 16.41 & $\ldots$ & 16.31 & 16.28 & $\cdots$ \\
\hline & 0.034 & 0.023 & 0.019 & 0.018 & 0.019 & 0.015 & 0.013 & 0.016 & 0.020 & $\ldots$ & 0.024 & 0.026 & $\ldots$ \\
\hline
\end{tabular}

